\section{Tegaserod therapy for chronic constipation}

Chronic constipation is a common problem, but effective treatments are lacking. A recent international study has shown that tegaserod, a selective $5-\mathrm{HT}_{4}$-receptor agonist, provides rapid and effective relief for constipation sufferers and is well tolerated.

The trial included 1,264 patients, the majority of whom were female, with at least a 6-month history of constipation (defined as fewer than three complete, spontaneous bowel movements per week, plus straining, a feeling of incomplete evacuation, and hard or very hard stools for at least a quarter of the time). Following a 2-week baseline period, patients were randomized to either $2 \mathrm{mg}$ or $6 \mathrm{mg}$ tegaserod twice daily, or placebo, during a double-blind, 12-week treatment phase. An increase in the number of complete, spontaneous bowel movements per week indicated a response to treatment.

Response rates during the first 4 weeks of treatment were $35.6 \%$ and $40.2 \%$ for patients receiving the $2 \mathrm{mg}$ and $6 \mathrm{mg}$ tegaserod doses, respectively, compared with $26.7 \%$ for the placebo group. For the higher dose of the drug, this response was maintained throughout the 12-week treatment period and was associated with significant improvements in abdominal pain or discomfort, and abdominal bloating or distension and straining.

Observing that these improvements were likely to be clinically as well as statistically significant, the authors conclude that tegaserod therapy is safe and efficacious in the treatment of chronic constipation.

Original article Kamm MA et al. (2005) Tegaserod for the treatment of chronic constipation: a randomized, double-blind, placebo-controlled multinational study. Am J Gastroenterol 100:362-372

\section{Tamoxifen and nonalcoholic fatty liver disease}

Tamoxifen treatment has been linked to an excess risk of nonalcoholic steatohepatitis, which is characterized by inflammation and fatty deposits in the liver. Bruno and colleagues have investigated this in a large group of women recruited into the multicenter Italian tamoxifen chemoprevention trial.
A total of 5,408 women aged $35-70$ years were randomized to tamoxifen ( $20 \mathrm{mg}$ daily) or placebo for a period of 5 years. All the women were healthy but had previously had a total hysterectomy to avoid endometrial cancer. Of the 5,087 participants who had a normal alanine aminotransferase level at baseline, 52 had elevated concentrations ( $\geq 1.5$ times the normal upper limit) on at least two occasions over a 6-month period, and tested negative for hepatitis $\mathrm{C}$ virus. Ultrasound findings indicated the presence of fatty liver in all these women. Of 20 women who subsequently underwent liver biopsy, 15 had steatohepatitis and 5 had fatty liver.

Suspected nonalcoholic fatty liver disease was significantly more common among women receiving tamoxifen than in those in the placebo arm. Analysis by BMI indicated that this association was restricted to those who were overweight or obese. Severe baseline hypercholesterolemia, hypertension, and BMI of $\geq 25 \mathrm{~kg} / \mathrm{m}^{2}$ were also revealed as independent predictors of the condition.

In summary, the study showed that tamoxifen doubled the risk of developing nonalcoholic steatohepatitis, but only in those women who were overweight or obese. This excess risk was limited to the first 2 years of intervention, however, and the absolute risk remained low. Bruno et al. comment that this information reduces the need for liver biopsy in women with elevated alanine aminotransferase levels during tamoxifen treatment.

Original article Bruno S et al. (2005) Incidence and risk factors for non-alcoholic steatohepatitis: prospective study of 5,408 women enrolled in Italian tamoxifen chemoprevention trial. BMJ [doi: 10.1136/bmj.38391.663287.E0]

\section{Chronic pancreatitis and quality of life}

Quality of life is increasingly viewed as an important issue in the management of chronic illness. A recent study from Italy has assessed the healthrelated quality of life of patients with chronic pancreatitis, showing that pain is the major factor affecting the well-being of these patients.

Pezzilli et al. recruited 190 patients with proven chronic pancreatitis of mean duration $201 \pm 141$ months. In the majority of cases $(77 \%)$, the condition was caused by alcohol 